\title{
EL PAPEL DE LOS CONTENIDOS PROCEDIMENTALES EN LA ADQUISICIÓN DEL CONOCIMIENTO EN EL ÁREA DE EDUCACIÓN FÍSICA
}

\section{Autores}

FERNANDO DEL VILLAR ÁLVAREZ* JOSÉ ANTONIO JULIÁN CLEMENTE**
DAMIÁN IGLESIAS GALLEGO* JUAN PEDRO FUENTES GARCÍA*

GRUPO DE INVESTIGACIÓN "ANÁLISIS DIDÁCTICO DELA ENSEÑANZA Y DEL RENDIMIENTO DEPORTIVO"

* FACULTAD DE CIENCIAS DEL DEPORTE. UNIVERSIDAD DE EXTREMADURA ** FACULTAD DE CIENCIAS DELA SALUD YEL DEPORTE. UNIVERSIDAD DE ZARAGOZA

\section{RESUMEN}

En el trabajo que presentamos exponemos una propuesta innovadora del tratamiento de los contenidos procedimentales en el área de educación física. Partiendo de la diferencia entre conocimiento declarativo y procedimental, es decir entre "saber" y "saber hacer", rompemos la clasificación tradicional existente en Educación Física, en la cual los contenidos procedimentales han estado ligados exclusivamente a las tareas motrices, reservando el tratamiento del conocimiento, dentro de los contenidos conceptuales.

Creemos que debe operarse un cambio en el tratamiento de los contenidos procedimentales en Educación Física, en tanto que existe un conocimiento procedimental, que está más cerca de los procedimientos (como ocurre en otras áreas del currículo escolar). Es por ello que proponemos el establecimiento de dos tipos de contenidos procedimentales, a saber, los contenidos procedimentales motrices y los contenidos procedimentales cognitivos.

Finalmente realizamos una propuesta de aplicación de los contenidos procedimentales cognitivos, a través de los juegos deportivos de invasión.

\section{PALABRAS CLAVES}

Contenidos procedimentales, Conocimiento, Iniciación deportiva.

\section{La organización del}

\section{conocimiento en Educación Física}

El carácter integral de la Educación Física nos aproxima a la búsqueda de objetivos en el plano motor, cognitivo, afectivo y social de nuestros alumnos, si bien el aprendizaje de las acciones motrices son el eje central y el objetivo de nuestra intervención educativa.

Las experiencias de movimiento que vive el alumnado en el aula de educación física se sustentan desde el diseño de situaciones motrices que proponen los profesores, tratando de cubrir objetivos dentro de una triple orientación formativa: la "educación en el movimiento", "educación a través del movimiento" y "educación acerca del movimiento" Arnold (1990).

Bajo esta perspectiva, los objetivos específicos de la Educación Física centran su atención en la adquisición de acciones motrices y en la comprensión de la práctica de las habilidades motrices. Es en este binomio aprendizaje motriz - comprensión del movimiento, donde situamos el papel del conocimiento en la educación física. El alumno adquiere conocimientos "acerca del movimiento" como medio para mejor comprender las acciones motrices que practica.

La adquisición del conocimiento en educación física debe por tanto convertirse en objetivo prioritario del profesorado, especialmente a partir de las investigaciones que definen la relación entre el conocimiento y el rendimiento deportivo, y que plantean que la adquisición de conocimiento es una condición previa para el aprendizaje de habilidades deportivas complejas (Anderson, 1976, 1982; Chi and Rees, 1983; Fischer, 1980; Wall, 1986, citado por Ruiz, 1995).

La organización del conocimiento en el área de Educación Física adquiere especial relevancia a partir de la importante distinción que Anderson (1982) establece entre conocimiento declarativo y conocimiento procedimental. Esta distinción que surge dentro de la Psicología Cognitiva nos sitúa en el centro del debate de los contenidos procedimentales, que este artículo desea plantear en su aplicación a la Educación Física. 
- El conocimiento declarativo es el conjunto de atributos y características que decimos de un objeto, un suceso o una idea. Es el concepto que tenemos de algo. Se identifica como "el saber", "saber decir", "saber qué".

En el área de educación física encontramos varios ejemplos:

$\checkmark$ Objeto - Concepto de balón medicinal, como un balón pesado que se utiliza para la mejora de la fuerza muscular.

$\checkmark$ Suceso-Concepto de juegos olímpicos, como la organización deportiva que cada 4 años celebra una competición multideportiva entre naciones.

$\checkmark$ Idea-Concepto de resistencia, como la capacidad de un ser humano para aguantar un esfuerzo físico durante un tiempo prolongado.

- El conocimiento procedimental es la forma en la que las personas utilizan o aplican su conocimiento declarativo. Se define como el conocimiento de cómo hacer las cosas. Implica la utilización coordinada de conceptos con un fin concreto. El ser humano realiza operaciones (manuales o cognitivas) en donde tiene que utilizar los esquemas conceptuales conocidos.

Se identifica como "el saber cómo", "saber hacer".

Ejemplos de educación física:

$\checkmark$ Resolver problemas tácticos-Proceso de toma de decisiones en una acción táctica, que tiene una respuesta motriz (tiro a canasta) y cognitiva (verbalización de la intención de la acción realizada).

$\checkmark$ Planificar una acción-Proceso de elaboración escrita de un plan para la mejora de la fuerza de las extremidades inferiores, o elaboración escrita de un encadenamiento gimnástico de forma individual o colectiva.

$\checkmark$ Aplicar nuevos conceptos-Redactar por escrito y realizar de forma práctica un calentamiento para el voleibol, aplicando los principios del calentamiento general, ya conocidos, o que analicen los puntos básicas para realizar un buen gesto de pase de dedos de voleibol.

\section{Los contenidos procedimentales en el curriculo escolar}

Con la implantación generalizada de los principios de la LOGSE en el sistema educativo, los contenidos escolares se ajustan definitivamente a una concepción integral de la formación del alumno. Los Diseños Curriculares de las diferentes materias incluyen una estructura de aprendizaje de contenidos en la línea de lo que hay que saber (conceptos), saber hacer (procedimientos) y ser (actitudes).
Los tres contenidos son objeto de aprendizaje, y por tanto los docentes de todas las áreas deben preocuparse por su adquisición. Los contenidos conceptuales y actitudinales han sido ampliamente desarrollados en la literatura educativa, especialmente a partir de la clasificación de Merrill (1983).

Los contenidos conceptuales, "el saber", son los que responden al conocimiento declarativo. Implica el aprendizaje de hechos, conceptos y principios sobre la motricidad humana, que son la base para la construcción del conocimiento procedimental. Éstos a su vez se dividen en:

Hechos

Conceptos

$\checkmark$ Sistemas conceptuales o principios

Los contenidos actitudinales responden al "ser" y se preocupan por los sentimientos o pensamientos que tenemos sobre los hechos, y que nos lleva a actuar de una forma determinada. Se concretan en:

$\checkmark$ Valores, conjunto de creencias personales.

$\checkmark$ Actitudes, predisposición a realizar determinadas actividades.

Normas, principios que guían nuestra conducta.

Por el contrario los contenidos procedimentales encuentran un menor nivel de concreción, y no existe acuerdo sobre su clasificación, dependiendo además de las diferentes áreas curriculares.

Entendemos los contenidos procedimentales como el conjunto de acciones ordenadas y dirigidas hacia un fin (Coll, 1986: Zabala, 1994). Esos contenidos presentan tres importantes características:

$\checkmark$ Implican una acción (saber hacer algo)

$\checkmark$ Responden a una secuencia ordenada y planificada (plan de acción inteligente)

$\checkmark$ Se realizan orientados a un objetivo (hacia un fin)

Por otro lado, los contenidos procedimentales pueden clasificarse en función de tres criterios (Zabala, 1994):

1. En función del lugar que ocupan en el continuum "motriz-cognitivo". Es decir un procedimiento implica más o menos cognitivamente al alumno. Los procedimientos pueden, en Educación Física, mostrar un predominio hacia lo motriz o hacia lo cognitivo, aun sabiendo que en nuestra área habitualmente presentan ámbos componentes. Para clarificar su comprensión podemos ejemplificar en Educación Física una secuencia que vaya desde la realización de una tarea motriz automatizada, un salto con pies juntos, en la que hay una escasa implicación cognitiva, pasando por la realización de un saque de voleibol, siguiendo por una acción individual de juego en baloncesto de $1 \times 1$, hasta una acción colectiva deportiva de $5 \times 5$.

2. En función del número de acciones que intervienen en la ejecución, representadas en el continuum "pocas acciones - muchas acciones". La elaboración 
de un plan de acción implica coordinar pocos elementos o muchos elementos. En el plano motriz iría desde las acciones donde se implican pocos grupos musculares, pocos segmentos corporales, un salto vertical, hasta las acciones de coordinación intermuscular e intersegmentaria, una entrada a canasta pasada con reverso. En el plano cognitivo, evolucionaría desde una sencilla elaboración de una lista de 5 ejercicios de flexibilidad de piernas, hasta un complejo plan de mejora de la flexibilidad para una temporada deportiva.

3. En función del grado de predeterminación del orden de la secuencia, representadas por el continuum "algorítmico-heurístico". La planificación de la secuencia de acción puede ser lineal, es decir que sigue una secuencia conocida y cerrada, con tareas de autorregulación, una voltereta adelante, - la secuencia puede ser abierta, por tanto imprevista, variable durante la propia realización de la acción, con tareas de regulación externa, una conducción de balón en fútbol. En el plano cognitivo. en Educación Física, evolucionaría desde la verbalización del alumno acerca de la colocación de la superficie de contacto en el saque de voleibol, hasta la situación donde verbaliza un plan de acción táctico para resolver una situación de superioridad en $3 \times 2$ en baloncesto.

\section{Hacia un nuevo tratamiento}

de los contenidos procedimentales

en el área de Educación Física

Tradicionalmente en Educación Física los contenidos procedimentales han estado ligados exclusivamente a las tareas motrices, reservando todos los aspectos de conocimiento a los contenidos conceptuales. Pero si entendemos que los contenidos conceptuales hacen referencia al conocimiento únicamente declarativo, tal como ocurre en otras áreas curriculares, creemos que debe operarse un cambio en el tratamiento de los contenidos procedimentales en Educación Física, en tanto que existe un conocimiento procedimental, que está más cerca de los procedimientos.

Aprender un contenido procedimental implica "saber hacer", pero bajo el binomio "aprendizaje motriz - comprensión del movimiento". El profesor de Educación Física, al objeto de desarrollar el "saber hacer" de su alumnado, deberá dedicar una parte de los contenidos procedimentales a que el alumno/a aprenda las conductas motrices, y otra parte de los contenidos procedimentales a que el alumno comprenda lo que hace motrizmente.

Nuestra propuesta de tratamiento de los contenidos procedimentales en Educación Física se basa en esta doble dimensión de "aprendizaje motriz - comprensión del movimiento". Por ello, partiendo de los criterios de clasificación de los contenidos procedimentales en el currículo escolar (Zabala,1994), desarrollaremos la aplicación a la Educación Física.
Los contenidos procedimentales se clasifican, tal como veíamos con anterioridad, en función de tres rasgos:

- La complejidad cognitiva del conocimiento elaborado.

- El número de elementos que debemos recordar para elaborar un plan de acción.

- El carácter cerrado o abierto del plan de acción diseñado.

De este modo creemos que en el tratamiento de los contenidos procedimentales en Educación Física parece más oportuno diferenciar entre:

1) contenidos procedimentales motrices, caracterizados porque

$\checkmark$ Requieren una menor implicación cognitiva.

$\checkmark$ Utilizan un plan de acción sencillo, con poca exigencia de combinación de elementos.

Utilizan un plan de acción lineal, fácilmente repetible.

2) contenidos procedimentales cognitivos, caracterizados porque:

$\checkmark$ Requieren una amplia implicación cognitiva.

$\checkmark$ Utilizan un plan de acción complejo, con gran exigencia de combinación de elementos cognitivos.

$\checkmark$ Utilizan un plan de acción heurístico, variable.

La aplicación de esta clasificación a la enseñanza deportiva nos remite, al debate entre el "modelo técnico" y el "modelo comprensivo", recordando que en el modelo técnico el docente no tiene interés porque sus alumnos adquieran conocimiento. Por el contrario en el modelo comprensivo, sí existe una preocupación por la adquisición del conocimiento, especialmente en los deportes colectivos, de forma específica a través de la "pedagogía de los modelos de decisión táctica" (Gréhaigne, 2001).

Por todo ello, creemos que el aprendizaje de los contenidos procedimentales en Educación Física se organiza en función de 4 tipos de actividades, complementarias entre sí, y que son la base para entender la iniciación deportiva:

a) Realización de la acción. Probar, experimentar sin un nivel de conciencia.

b) Automatización de la acción. Repetir la acción hasta que se automatiza, se interioriza en los esquemas motores.

c) Reflexión sobre la acción. Tomar conciencia de las características de la acción realizada y de la secuencia seguida. Es el aprendizaje comprensivo del deporte, lo cual implica comprender la acción deportiva que realizamos.

d) Aplicar la acción en nuevos contextos. Al automatizar la parte técnica de la acción y comprender sus características, debemos aplicarla en contextos nuevos, desarrollando una acción táctica propia, dado que varían los elementos del entorno, y las situaciones no son previsibles. 


\section{Los contenidos procedimentales cognitivos. Una aplicación a la enseñanza de los juegos deportivos de invasión}

En el proceso de iniciación deportiva el papel del conocimiento es determinante. Gréhaigne (2001) establece que "en la formación de un deportista es indispensable que éste tome conciencia de las condiciones de éxito que permiten la interpretación y la explicación de la acción (pp 149).

El alumno bajo el enfoque comprensivo de la enseñanza del deporte (Almond, 1986; Bunker y Thorpe, 1982; Devis, 1992), también llamado "pedagogía de los modelos de decisión táctica" (Gréhaigne, 2001) debe comprender la naturaleza del juego a través de situaciones motrices que incluyan los elementos estructurales del deporte pero simplificándose sus condiciones de práctica, a través de los llamados "juegos modificados" (Devís, 1992: Thorpe, Bunker and Almond, 1986).

En el proceso de enseñanza de los deportes, esta distinción de tipos de conocimiento tiene una especial relevancia a la hora de establecer qué modelo pedagógico utilizaremos para enseñar las habilidades deportivas.

De este modo, el "modelo técnico", "la pedagogía de los modelos de ejecución" (Goirand, 1987) prioriza la enseñanza aislada de las habilidades motrices, presentadas fuera del contexto real de juego. El conocimiento debe orientarse en este modelo hacia el "conocimiento declarativo"de las razones empíricas del movimiento. El profesor debe proporcionar información científica, basada en fundamentos biomecánicos, acerca de la técnica deportiva.(Arnold, 1990; Kirk, 1990)

Por el contrario, el "modelo comprensivo" o "pedagogía de los modelos de decisión táctica" (Gréhaigne, 2001) prioriza la enseñanza en situaciones reales de juego de los elementos tácticos. Thorpe, Bunker and Almond (1986) plantean que el aprendizaje de la conciencia táctica debe ser anterior al aprendizaje específico de las destrezas técnicas. Bajo este enfoque el "conocimiento procedimental" aparece como prioritario, y debe centrarse en la enseñanza del conocimiento táctico de los principios del juego y en el desarrollo de la capacidad para tomar decisiones en situación real de juego (juegos modificados).

Parece, por tanto, que la iniciación deportiva debe basarse en el «juego modificado», dado que esta situación global de aprendizaje deportivo cumple perfectamente dos principios fundamentales:

$\checkmark$ Mantienen la estructura básica de los deportes, pues poseen reglas y principios tácticos similares.

$\checkmark$ Se adaptan a las características motrices y cognitivas de los escolares en sus diferentes etapas evolutivas.
El juego modificado permite iniciar la enseñanza del deporte, desde la propia situación del juego real. El niño comienza compitiendo (jugando), pero en situaciones reducidas que le permiten una mejor comprensión del juego deportivo, creando la necesidad de aprender nuevos elementos, para solucionar problemas acaecidos en el juego.

El educador debe adaptar las reglas, reducir/aumentar el espacio y el número de componentes, modificar los materiales, pero manteniendo la misma estructura de los principios de cooperación - oposición.

Graça y Oliveira (1997) refuerzan esta idea, concretando que los juegos modificados deben cumplir los siguientes requisitos:

$\checkmark$ Preservar la autenticidad del juego, respetando el concepto básico del deporte.

$\checkmark$ Contemplar los elementos estructurales esenciales del juego real.

Tener siempre presentes las relaciones de cooperación-oposición.

$\checkmark$ Establecer una dinámica de juego en el que la alternancia de ataque - defensa sea natural.

$\checkmark$ No condicionar la actividad de los alumnos a respuestas cerradas

Creemos que los juegos modificados representan un buen instrumento de aprendizaje, pero a la vez pensamos que en la enseñanza deportiva debemos utilizar estrategias didácticas complementarias para desarrollar los contenidos procedimentales cognitivos (el aspecto decisional de la toma de decisiones), y que tan importante es de cara a optimizar las conductas de nuestro alumnado en la acción deportiva.

El aprendizaje de las conductas táctico-técnicas, en el juego bajo el modelo comprensivo, se basan fundamentalmente en el conocimiento procedimental, o lo que es lo mismo, en que el alumno comprenda lo que se está haciendo cuando desarrolla las acciones técnicas o instrumentos (bote, pase, lanzamiento, etc.) dentro del juego.

Del mismo modo, se trata de aplicar la teoría del esquema motor de Schmidt (1975) relativa al modelo de aprendizaje basado en la práctica variable. Es decir, que una persona que ha aprendido un gesto técnico o instrumento, en unas condiciones de enseñanza basada en la práctica variable (práctica en diferentes desplazamientos, uso de diversos móviles, en cuanto a su peso, a la capacidad de bote, etc.) de los elementos principales que determinan su adquisición, tienen mayores posibilidades de comprender lo que está haciendo, de perfeccionarlo y de transferir el procedimiento a nuevas situaciones.

Por lo tanto debemos asegurarnos que el sujeto posea un amplio bagaje motor de habilidades básicas, adquirido en situaciones de variabilidad de la práctica, de cara a asegurarnos un óptimo aprendizaje de los saberes fundamentales del contenido (llevar la iniciativa tanto en ataque como en defensa, los principios generales del juego, las responsabilidades individuales de defensa, las conductas táctico-técnicas individuales y colectivas básicas, etc.) que estamos intentando enseñar. 
Nuestra propuesta para la valoración de saberes fundamentales en los juegos deportivos de invasión, como son las conductas táctico-técnicas individuales y las conductas colectivas básicas, pasa por la confección de material curricular específico, denominadas "representaciones gráficas del conocimiento procedimental".

La justificación de la incorporación de este material al proceso de enseñanza-aprendizaje, se centra sobre los siguientes aspectos. A saber:

1. Las "representaciones gráficas del conocimiento procedimental" son un tipo de práctica concreta a trabajar en la enseñanza de los juegos deportivos. En la literatura especializada se entiende como práctica a "los distintos procedimientos o técnicas utilizadas para manejar más eficazmente esa información con el objeto de producir aprendizaje" (Oña, Martínez, Moreno y Ruiz, 1999, pp. 206). Y si nos disponemos a solucionar situaciones concretas de las que nos referimos estamos fomentando la reorganización de la información que el sujeto tiene sobre una situación concreta de juego.

2. Inciden directamente en ciertos componentes del conocimiento estratégico. Entre los que vamos a destacar dos; el metaconocimiento y los contenidos conceptuales específicos.

Sobre la metacognición, porque activa principalmente dos tareas cognitivas esenciales, la planificación y selección de la acción, y la evaluación de la respuesta elegida. La primera se realiza de forma privada o individual, cumplimentando las representaciones planteadas, mientras que la segunda se realiza de forma colectiva en los debates realizados durante las sesiones.

Y sobre los conocimientos temáticos específicos, porque la respuesta estará en función de una serie de aspectos que deberá conocer, como son las reglas del juego, los elementos formales del juego deportivo, el conocimiento de los principios generales del juego, el conocimiento de los principios tácticos individuales y colectivos básicos, etc. De su conocimiento dependerá en gran parte una u otra opción de respuesta y su posterior justificación.

3. Permite conocer el nivel de conocimiento que tiene el alumnado sobre las conductas tácticas del juego. De forma que no sólo lo evaluemos por su ejecución, que es la conducta observable del acto motor, si no también por su capacidad de decidir lo más adecuado en una situación determinada, valorando del mismo modo la justificación de la misma.

Otro de los aspectos a considerar son las funciones de cara al proceso de aprendizaje. Funciones que no deben ser entendidas como aisladas, sino que intentaremos utilizarlas a lo largo del proceso de enseñanza-aprendizaje. Principalmente podemos destacar tres. A saber:

1. Como elemento complementario a las explicaciones docentes. Cuando estamos introduciendo la sesión o durante la puesta en común del final de la misma, podemos presentar algunas situaciones para debatir o explicar.

2. Como elemento de control y avance del aprendizaje. Y es que compartimos la idea de Pozo (1996) al comentar que "los verdaderos sistemas de evaluación del aprendizaje deberían implicar un control continuo de las cambios que se producen, o al menos alguna medida de las diferencias entre el punto de partida del aprendiz (sus conocimientos anteriores) y el punto de llegada tras la instrucción (los nuevos conocimientos).

3. Como instrumento de evaluación. Diseñamos situaciones concretas para que cumplan la función de evaluar al alumnado. Somos partidarios de incluirla como una información más, dentro de una evaluación formativa del alumnado. (Ramos, Del Villar y Moreno, 1998).

En conformidad con lo anterior, creemos conveniente reflexionar sobre los momentos en los que puede ser utilizado a lo largo de una sesión de Educación Física. Podemos destacar los siguientes:

a) Al comienzo de la sesión. El docente en la información inicial de la sesión, puede utilizar una o dos situaciones en la pizarra, para que pueda ser resuelta de forma colectiva. Esto crea un debate sobre lo que estamos aprendiendo y sirve para centrar la atención en los aspectos claves que el alumnado debe prestar la atención.

b) Al final de la sesión de forma colectiva. Durante la reflexión final de la sesión, podemos usarlo como debate de ciertos comportamientos que han tenido lugar en el desarrollo de la puesta en práctica de situaciones. Utilizaríamos la pizarra como elemento de referencia.

c) Al final de la sesión de forma individual. El tiempo de reflexión del final de clase es utilizado por el docente, para comentar aspectos generales de la sesión (comportamientos, sucesos, etc.) y posteriormente para repartir unos folios con situaciones táctico-técnicas colectivas básicas para resolver de forma individual.

d) Al final de la sesión de forma individual-colectiva. Complementando la anterior situación, lo que se haría posteriormente sería comentar las respuestas de los alumnos/as a las tareas problema que hemos propuesto.

El material curricular diseñado para estas representaciones posee varias partes. Un enunciado, donde el alumno/a se identifica con uno de los roles de juego (jugador atacante con balón, jugador atacante sin balón, defensor del jugador atacante con balón, defensor del jugador atacante sin balón) y una acción a realizar. La representación gráfica, con la dirección del ataque, los elementos formales del juego y la distribución de los jugadores. Finalmente aparece un cuadro para justificar la respuesta elegida.

A continuación presentamos cuatro situaciones, una por cada rol de juego (del dos contra dos), que ejemplifican todo lo comentado hasta el momento. 
Estás jugando a un deporte de invasión, ¿Qué desplazamiento realizarías para conseguir pasar con más facilidad la pelota a tu compañero? Señala con una flecha el recorrido que harías. Justifica la respuesta en el recuadro en blanco.
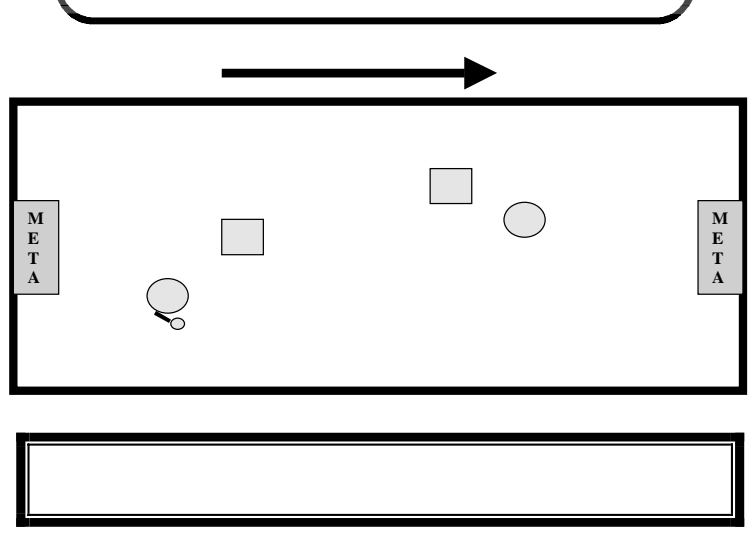

Figura 1

Ejemplo de "representaciones de valoración táctico-técnica" de jugador atacante con balón.
Estás jugando a un deporte de invasión, ¿dónde te colocarías para recibir mejor la pelota de tú compañero? Señala con una flecha el recorrido que harías. Justifica la respuesta en el recuadro en blanco.
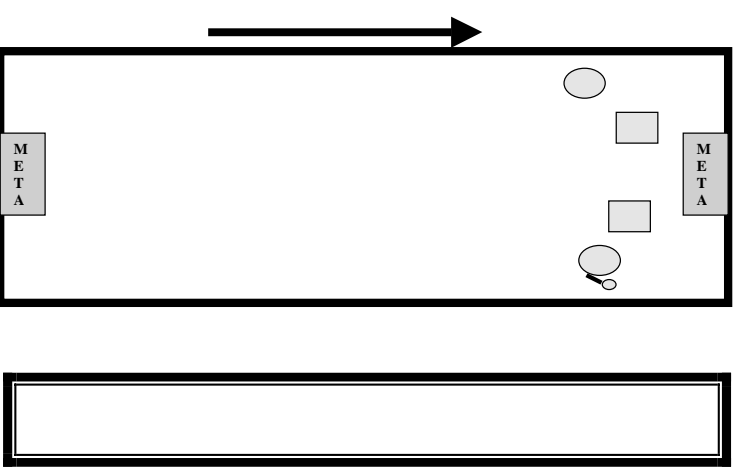

\section{Figura 2}

Ejemplo de "representaciones de valoración táctico-técnica" de jugador atacante sin balón.
Estás jugando a un deporte de invasión, ¿dónde te colocarías para defender mejor la situación de ataque del gráfico?. Dibuja un cuadro señalando el lugar dónde te pondrías. Justifica la respuesta en el recuadro en blanco.
Estás jugando a un deporte de invasión, ¿dónde te colocarías para defender mejor la situación de ataque del gráfico?. Dibuja un cuadro señalando el lugar dónde te pondrías. Justifica la respuesta en el recuadro en blanco.
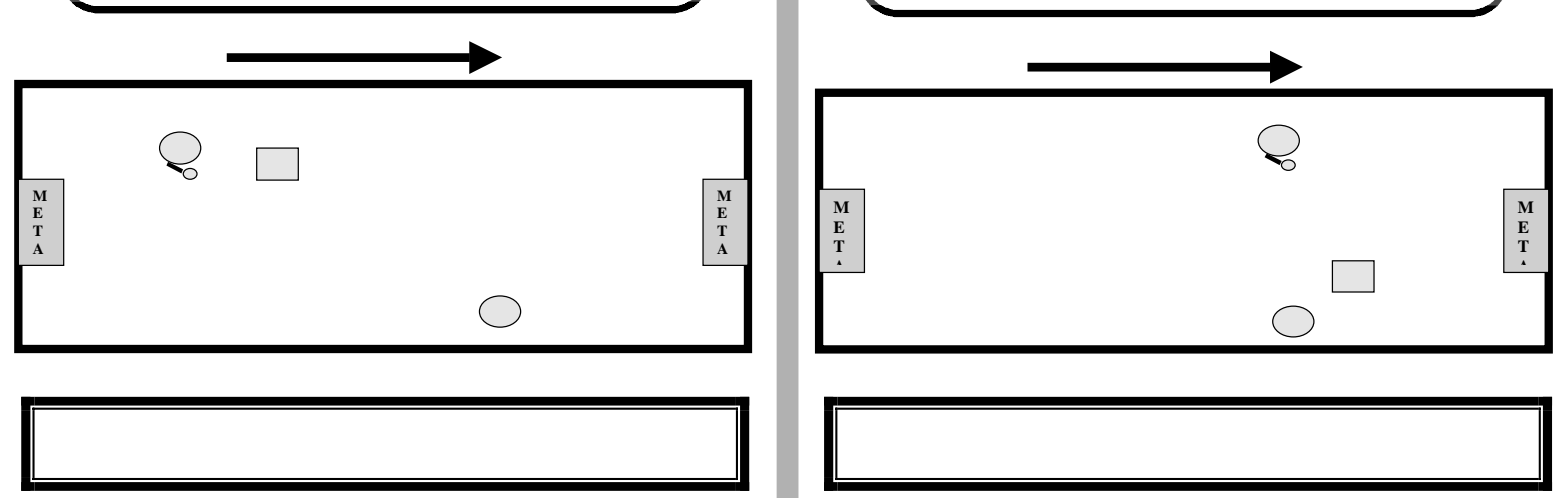

Figura 3.

Ejemplo de "representaciones de valoración táctico-técnica" de defensor del jugador atacante con balón.
Figura 4.

Ejemplo de "representaciones de valoración táctico-técnica" de defensor del jugador atacante sin balón. 


\section{Consideraciones finales}

En el presente artículo hemos realizado una revisión del tratamiento de los contenidos procedimentales en la clase de Educación Física y hemos planteado una propuesta alternativa para el trabajo de los contenidos procedimentales cognitivos. Hemos incluido la visión reflexiva y comprensiva del movimiento, de cara a reforzar el modelo de desarrollo motor basado en la noción de conocimiento (Wall y col. 1985; Wall, 1986; en Ruiz, 1995).

La utilización de las representaciones gráficas para la elaboración de las conductas táctico-técnicas, durante el proceso de enseñanza-aprendizaje de los juegos deportivos, es una estrategia didáctica complementaria a la práctica motriz. Pero, que sin duda, en un futuro tendremos que demostrar empíricamente si favorece la adquisición de conductas táctico-técnicas optimas durante el juego. Del mismo

\section{Referencias bibliográficas}

Almond, L. (1986). Reflecting on themes: a games classification. In R. Thorpe, D. Bunker, y L. Almond (Eds.) Rethinking games teaching. Loughborough, U. K.: University of Technology, Departament of Physical Education and Sport Science.

Anderson, J. R. (1976). Language, memory and thought. Hillsdale, NJ: Erlbaum.

Anderson, J.R. (1982). Acquisition of cognitive skill. Psychological Review, 89, 369-406.

Arnold, P.J. (1990). Educación Física, movimiento y curriculum. Madrid: Morata, pp.127-139.

Bunker, D.J. y Thorpe, R.D. (1982). A model for the teaching of games in secondary schools. The Bulletin of Physical Education, 18, 9-16.

Chi, M. T. H., y Rees, E. T. (1983). A learning framework for development: The control and construction of hierarchies of skills. Psychological Review, 87, 447-531.

Coll, C. (1986). Marco curricular para la enseñanza obligatoria. Barcelona: Departament d'Ensenyament de la Generalitat de Catalunya.

Devis, J. (1992). Nuevas perspectivas curriculares en educación física: La salud y los juegos modificados. Barcelona: INDE, pp. 141-160.

Fischer, K. W. (1980). A theory of cognitive development: The control and construction of hierarchies of skills. Psychological Review, 87, 477-531.

Goirand, P. (1987). Une problématique complexe: des practiques sociales des A.P.S. aux contenus $d^{\prime}$ enseignament en E.P.S. Spirales I, 7-38. modo, y en consonancia con la anterior aseveración, tendremos que investigar si estos procedimientos tienen utilidad para mejorar la adquisición del conocimiento en el alumnado.

Igualmente, otra de las cuestiones que pueden ser relevantes en el estudio de la actuación docente, es indagar cuál es el momento o momentos más adecuados para la introducción en la clase de Educación Física, de los recursos aquí presentados, y que sean capaces de producir un mayor aprendizaje en el alumnado.

Por todo ello, se abre un nuevo planteamiento del tratamiento de los contenidos procedimentales cognitivos, como instrumento para la mejora de la comprensión en el deporte, y como consecuencia para el aprendizaje de tareas motrices.

Graca, A. y Oliveira, J. (1997) - La enseñanza de los juegos deportivos. Barcelona: Paidotribo.

Gréhaigne, J.F. (2001). La organización del juego en el fútbol. Barcelona: INDE, pp. 141-150.

Kirk, D. (1990). Educación física y currículo. Valencia: Servicio de Publicaciones de la Universidad, pp. 80-91.

Merrill, M.D. (1983). Component display Theory, in Ch.M. Reigelhut (Ed.) Instruccional design: Theories and models. An overview of their current status. New Jersey: Hillsdale.

Oña, A., Martínez, M., Moreno, F. y Ruiz, L. M. (1999): Control y Aprendizaje Motor. Madrid: Síntesis.

Pozo, I. (1996). Aprendices y maestros. La nueva cultura del aprendizaje. Madrid: Alianza Editorial.

Ramos, L., Del Villar, F. y Moreno, P. (1998). La evaluación formativa en el proceso de formación del profesor de educación física. Revista de Educación Física. 70 5-10.

Ruiz, L. M. (1995). Competencia motriz. Elementos para comprender el aprendizaje motor en educación física escolar. Madrid: Gymnos.

Schmidt, R. A. (1975): A schema theory of discrete motor skill learning. Psychological Review, 82, 225-260

Thorpe, R., Bunker, D., y Almond, L. (1986). Rethinking games teaching. Loughborough, U. K.: University of Technology, Departament of Physical Education and Sport Science.

Zabala, A. (Coord.) (1994). ¿Cómo trabajar los contenidos procedimentales en el aula? Barcelona: Graó. 